\title{
CD40 expression in HCV-associated chronic liver diseases
}

\author{
KATSUYA SHIRAKI, KAZUSHI SUGIMOTO, HIROSHI OKANO, HIDETAKA WAGAYAMA, \\ KATSUHIKO FUJIKAWA, TAKENARI YAMANAKA, TAKESHI ITO, SHIGERU OHMORI, \\ TAKAHISA SAKAI, KOUJIRO TAKASE and TAKESHI NAKANO
}

First Department of Internal Medicine, Mie University School of Medicine, Tsu, Mie 514-8507, Japan

Received January 31, 2006; Accepted April 28, 2006

\begin{abstract}
CD40 is expressed primarily on B cells and plays an important role in antigen presentation, B cell proliferation, and $\mathrm{T}$ cell activation. It has been reported that the CD40 signal modulates apoptosis and has an anti-viral effect in certain cells. Therefore, we investigated the expression and the function of CD40 in HCV-associated chronic liver disease. The expression of CD40 on liver tissues was determined through immunohistochemistry on 50 liver specimens obtained from HCV-positive patients. The effect of CD40 signaling on apoptosis of HepG2 cells was assessed using the MTT assay. The effect of CD40 stimulation on NF- $\kappa \mathrm{B}$ activation was determined in $\mathrm{NF}-\kappa \mathrm{B}$ reporter gene-transfected HepG2 cells with the Luciferase assay. CD40 positive hepatocytes were observed in both periportal and lobular areas, accompanied by inflammation. In both areas, CD40 staining intensity became significantly stronger, correlating with the histological grading. Similarly, it became stronger with the progression of the histological staging in F1, F2 and F3 cases; however, the expression level decreased in F4 cases. CD40 ligation induced apoptosis in HepG2 cells in the presence of $500 \mathrm{ng} / \mathrm{ml}$ of actinomycin D, while CD40 ligation alone could not. Anti-CD40 monoclonal antibody caused NF- $\mathrm{\kappa B}$ activation in HepG2 cells in a dosedependent manner. These results suggest that hepatocyte overexpression of CD40 might play an important role in regulating hepatocyte survival and death in $\mathrm{HCV}$-associated chronic liver diseases.
\end{abstract}

\section{Introduction}

Tumor necrosis factor receptor (TNFR)-mediated apoptosis plays an important role in the host's defense against hepatocytes in chronic liver diseases by $\mathrm{T}$ lymphocytes (1-3). A previous study revealed that Fas antigen expression plays an important role in inflammation of the hepatitis $\mathrm{C}$ virus-infected liver, particularly in active inflammation of chronic hepatitis $C$ (4).

Correspondence to: Dr Katsuya Shiraki, First Department of Internal Medicine, Mie University School of Medicine, 2-174 Edobashi, Tsu, Mie 514-8507, Japan

E-mail: katsuyas@clin.medic.mie-u.ac.jp

Key words: CD40, HCV, chronic liver diseases
Another TNFR family member, CD40, a 48.000-m.w. transmembrane glycoprotein which does not contain death domains, was first identified and functionally characterized on B cells (5). CD40 plays a crucial role in the survival, proliferation, and differentiation of $\mathrm{B}$ cells. Cross-linking CD40 with either the CD40 ligand (CD40L) or anti-CD40 antibody rescues the germinal center and mature $\mathrm{B}$ cells from apoptotic cell death. CD40 may provide B cells with an important signal to rescue them from the selective pressure of Fas-mediated apoptosis (6).

Recent studies revealed that CD40 is also expressed by hematopoietic progenitors, endothelial cells, antigen-presenting cells and epithelial cells. These studies suggested that CD40 stimulation is essential for the initiation of antigen-specific $\mathrm{T}$ cell responses, autoimmunity, as well as $\mathrm{T}$ cell and macrophage activation. Furthermore, some carcinoma cell lines originating from bladder and mammary glands have been demonstrated to have CD40 expression on their cell surface (7). However, the functional role of CD40 expression has not been well elucidated in a variety of cells.

We have previously reported that HCC cell lines constitutively expressed CD40 mRNA and membrane bound CD40 antigen, which was upregulated slightly by interferon- $\gamma$ (8). In addition, $60 \%$ of human HCC tissues demonstrated positive CD40 staining, whereas non-tumor tissues showed little detectable staining. In HepG2 cells, CD40 stimulation does not affect cell viability, but significantly inhibits Fas and TNF receptor-mediated apoptosis in a dose-dependent manner by blocking the activation of CPP32. Furthermore, we concluded that CD40 expression in HCCs plays an important role in tumor biology, especially the resistance against Fas and TNF receptor-mediated apoptosis (8). It has been revealed that CD40L produces a wide range of growth-regulatory effects on CD40-expressing cells $(9,10)$. In some lymphomas and leukemia cells, such as HCC, CD40 activation contributed to tumor survival and resistance to chemotherapy (11). However in other lymphoma cells, such as Daudi B lymphoma cells, CD40 cross-linking induced cell cycle arrest, which was critical for the induction and maintenance of tumor dormancy (12). These results enhance the significance of CD40 expression in tumor cells (9).

Although CD40 is not expressed in normal liver tissue except Kupffer and endothelial cells, CD40 expression in chronic liver disease has not been well elucidated. Therefore, we investigated the expression of CD40 in HCV-associated chronic liver disease. We also examined the potential role of 

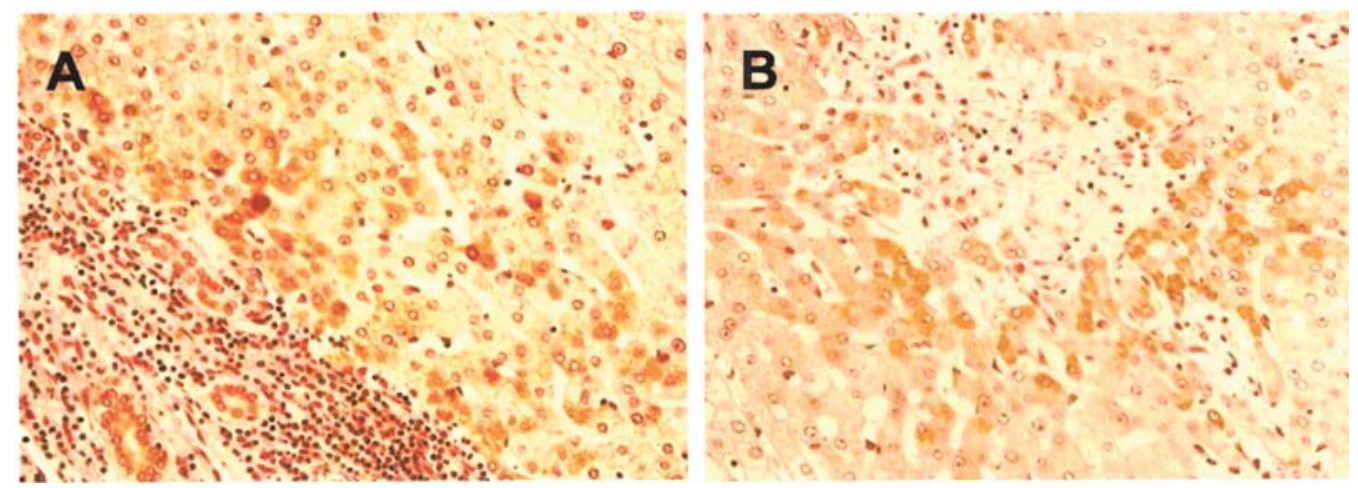

Figure 1. Immunohistochemical staining of human HCV-associated chronic hepatitis with anti-CD40 antibody. (A) CD40 expression is prominent in the periportal (B) and lobular area. CD40 is detected in both the cytoplasm and cell membrane.
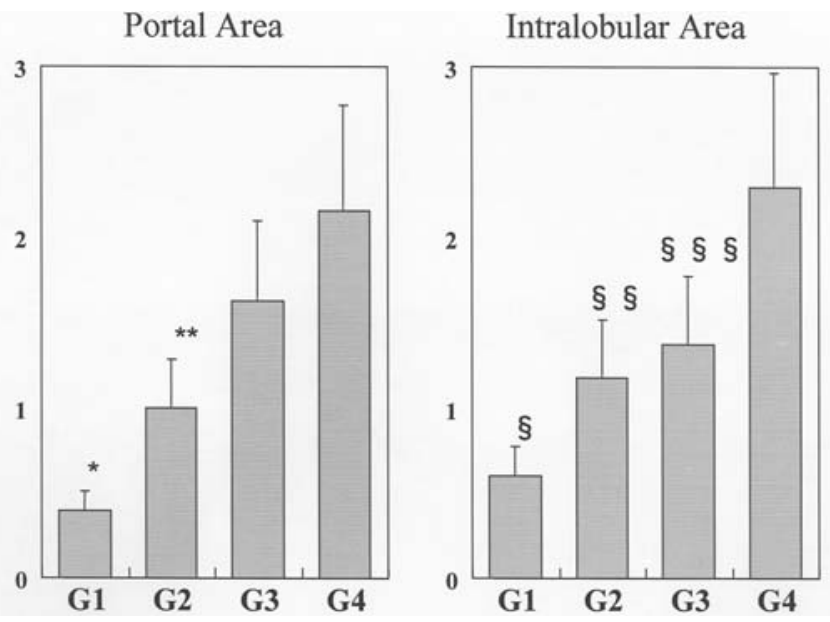

Figure 2. CD40 expression according to the degree of grading. CD40 expression was semi-quantified according to the scoring system. $\left({ }^{*} \mathrm{p}<0.01\right.$ vs. $\mathrm{G} 2, \mathrm{G} 3 ;{ }^{* *} \mathrm{p}<0.05$ vs. $\mathrm{G} 3 ; \mathrm{p}<0.01$ vs. $\mathrm{G} 4 ;{ }^{\circledR} \mathrm{p}<0.05$ vs. $\mathrm{G} 3 ; \mathrm{p}<0.01$ vs. $\mathrm{G} 4$; ${ }^{\S} \mathrm{p}<0.01$ vs. $\mathrm{G} 4 ;{ }^{\S \S} \mathrm{p}<0.01$ vs. G4).

CD40 signaling in cell proliferation as well as Fas and TNFRmediated apoptosis.

\section{Materials and methods}

Materials. The expression of CD40 in liver tissues was determined through immunohistochemistry on 50 liver specimens obtained from HCV-positive patients. Among these, 43 had chronic hepatitis and 7 had liver cirrhosis. All patients were diagnosed with $\mathrm{HCV}$-associated chronic liver disease by histological analysis, as well as HCV antibody detection (anti-HCV) in serum using the third-generation enzyme-linked immunosorbent assay (Abott Labs, IL) and HCV-RNA by reverse transcriptase-polymerase chain reaction. The patients who had HBs antigen or whose final diagnosis was hemochromatosis, autoimmune liver disease, primary biliary cirrhosis or Wilson's disease were excluded. Patients who developed hepatocellular carcinoma (HCC) within six months were also excluded from this study.

Tissue sections were stained with hematoxylin and eosin, and the stage of liver fibrosis and grade of liver activity were evaluated. The histological findings were sub-classified into five groups based on the stage of liver fibrosis according to
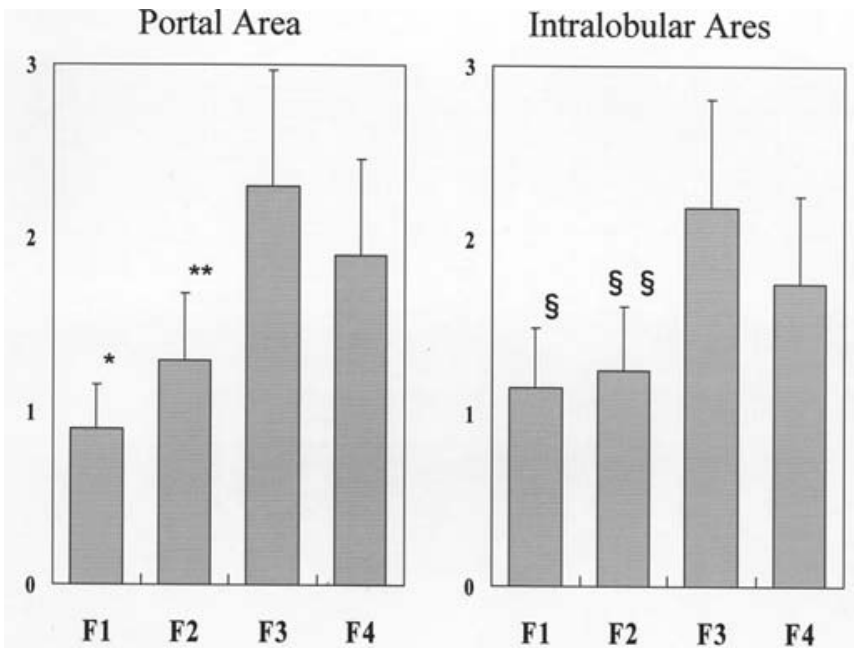

Figure 3. CD40 expression according to the degree of staging. CD40 expression was semi-quantified according to the scoring system. ( ${ }^{*} \mathrm{p}<0.01$ vs. G3; p<0.05 vs. G4; ${ }^{* *} \mathrm{p}<0.01$ vs. G4; ${ }^{\S} \mathrm{p}<0.01$ vs. G4; ${ }^{\S \S} \mathrm{p}<0.05$ vs. G4).

the Metavir classification: F0, no fibrosis; F1, portal fibrosis without septa; F2, portal fibrosis with few septa; F3, numerous septa without cirrhosis; and F4, cirrhosis. The grade of liver activity was classified into four groups using the Metavir classification: A0, no activity; A1, mild activity; A2, moderate activity; and A3, severe activity.

Immunohistochemical staining of CD40. Immunohistochemical staining for CD40 was performed on surgically resected HCC tissues, biopsy specimens of chronic hepatitis and normal liver using a labeled streptavidin-biotin method (Dako Corp., Carpinteria, CA). Deparaffinized sections were heated for 5 min at $100^{\circ} \mathrm{C}$ in a pressure cooker to reactivate the antigen and treated with $0.3 \% \mathrm{H}_{2} \mathrm{O}_{2}$ in methanol for $30 \mathrm{~min}$ to abolish endogenous peroxidase activity. Sections were blocked with $1 \%$ goat serum in PBS, covered with rabbit anti CD40 polyclonal antibody (C-20) (Santa Cruz Biotechnology, Inc.) (1:100 diluted in PBS) overnight at $4^{\circ} \mathrm{C}$, washed, covered with a second step biotinylated antibody for $30 \mathrm{~min}$, and incubated with peroxidase-labeled streptavidin for $30 \mathrm{~min}$. After washing, sections were incubated with $0.05 \%$ diaminobenzidene/ $0.15 \% \mathrm{H}_{2} \mathrm{O}_{2}$ and counterstained with $10 \%$ hematoxylin (Wako Pure Chemical Industries, Osaka, Japan). 


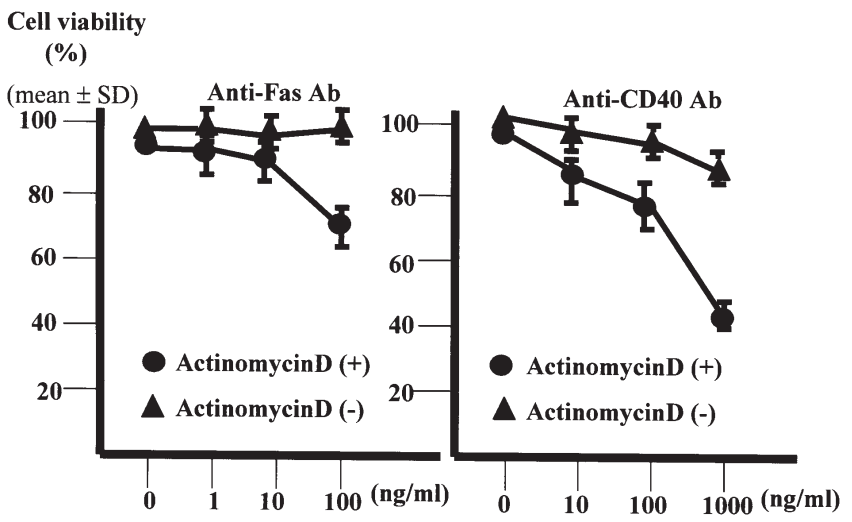

Figure 4. Effect of CD40 stimulation on cell viability in HepG2 cells. Cells were cultured with the indicated concentration of anti-Fas antibody in the presence or absence of $0.5 \mu \mathrm{g} / \mathrm{ml}$ of actinomycin D for $24 \mathrm{~h}$.

The degree of CD40 antigen expression in the periportal area was classified into four groups: 0 , none; 1 , positive cells in $<1 / 3$ of the hepatocytes in the circumference of most of the portal or lobular areas; 2 , involvement of $1 / 3-2 / 3$ of the hepatocytes in the circumference of the majority of the portal or lobular areas; and 3 , involvement of $>2 / 3$ of the hepatocytes in the circumference of most of the portal or lobular areas.

Detection of apoptosis. To assess the viability of HepG2 cells, the 3-(4,5-dimethylthiazol-2-yl)-2,5-diphenyl tetrazolium bromide (MTT) assay was performed. Briefly, 5x10 cells were cultured in each well of 96-well, flat-bottom microtiter plates (Corning Glass Works, Corning, NY), and incubated for $24 \mathrm{~h}$, at $37^{\circ} \mathrm{C}$ in $5 \% \mathrm{CO}_{2}$. Each reagent was added to the individual culture wells, $0.5 \mu \mathrm{g} / \mathrm{ml}$ actinomycin D (Sigma Chemical Co.) or $1 \mu \mathrm{g} / \mathrm{ml}$ anti-CD40 monoclonal antibody (BL-C4) (Caltag Laboratories), and incubated for $24 \mathrm{~h}$. Then, the viable cell count was assayed using the Cell Titer 96 assay kit (Promega, Madison, WI) according to the manufacturer's instructions. The absorbance of each well was measured with a microculture plate reader (Bio-Rad Laboratories, Tokyo, Japan) at $570 \mathrm{~nm}$. To confirm apoptotic cells, nuclei of cells were stained with 4'6,-diamidino-2phenylindole (DAPI) (Sigma Chemical Co.). The percentage of apoptotic cells was calculated as the percentage of apoptotic nuclei (nuclear condensation or nuclear fragmentation) in total nuclei ( 5000 nuclei) under a fluorescence microscope.

$N F-\kappa B$ luciferase reporter gene assay. The pNF-кB-Luc Vector (Mercury $^{\mathrm{TM}}$ pathway profiling system) was obtained from Promega. Human HCC cells $\left(2 \times 10^{5}\right)$ were grown in six-well plates in triplicate the day before transfection. Cells were transfected using FuGENE 6 (Boehringer Mannheim, Mannheim, Germany) and incubated for $18 \mathrm{~h}$ at $37^{\circ} \mathrm{C}$. The medium was then removed and cells were incubated in complete media for $24 \mathrm{~h}$. Cells were stimulated with recombinant human TRAIL (R\&D Systems) for the final $24 \mathrm{~h}$. Luciferase activity was determined from cell extracts by means of a luciferase assay system (Promega) and a luminometer (Berthold Analytical Instruments, Nashua, NH). The results are presented as the

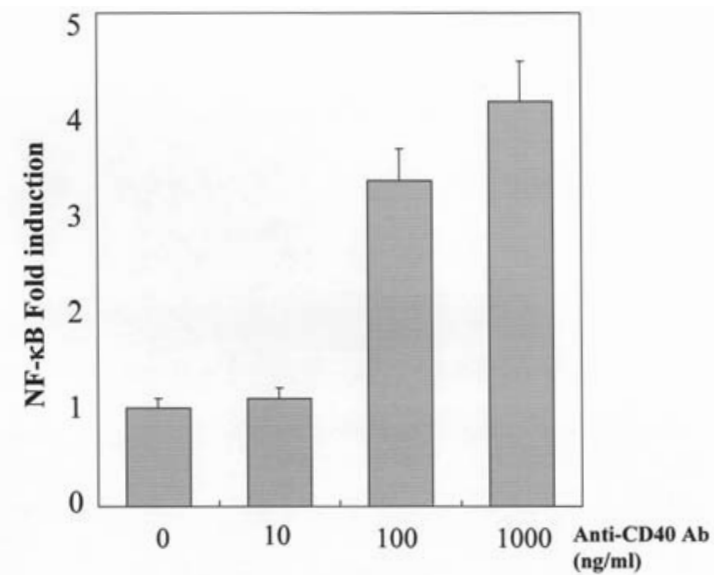

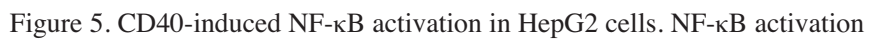
was analyzed using a NF-кB-luciferase reporter gene assay. The results are presented as fold induction of the luciferase activity observed in cells incubated with the indicated concentration of anti-CD40 monoclonal antibody. The data shown are the mean \pm SEM of three independent experiments.

fold induction above the luciferase activity found in cells without stimulation.

Statistical analysis. Results are expressed as mean \pm SD. The CD40 expression scores were analyzed by the MannWhitney test. A value of $\mathrm{p}<0.05$ was considered statistically significant.

\section{Results}

Expression of CD40 in HCV-associated chronic liver tissues. CD40 antigen was stained mainly in hepatocytes close to the portal (Fig. 1A) area and around areas of necrosis (Fig. 1B). At the cellular level, CD40 staining was observed in both the cytoplasm and cell membrane. We then scored CD40 expression in each periportal and lobular area. We analyzed mean CD40 expression scores according to grade of inflammation and stage of fibrosis. The results are shown in Fig. 2. CD40 scores increased according to grades of inflammation in both periportal and lobular areas (Fig. 2). However, CD40 scores did not correlate well with stages of fibrosis in either the periportal or lobular area, although CD40 scores were relatively high in advanced stage fibrosis (Fig. 3).

Effect of CD40 on cell viability in HepG2 cells. In order to determine the function of hepatocyte CD40 expression, we next investigated the effect of CD40 stimulation on cell viability on HepG2 cells. To stimulate CD40 on HepG2 cells, we used IgM type anti-CD40 monoclonal antibody, which was known to have an agonistic effect, since trimerization of CD40 is important for the transmission of its signal. CD40 stimulation alone by anti-CD40 antibody at a dose of $\leq 1 \mu \mathrm{g} /$ $\mathrm{ml}$ showed little effect on cell viability (Fig. 4). However, in the presence of $0.5 \mu \mathrm{g} / \mathrm{ml}$ actinomycin $\mathrm{D}$, a metabolic inhibitor, CD40 stimulation significantly decreased cell viability in a dose-dependent manner. Cells treated with antiCD40 antibody in the presence of actinomycin D showed typical apoptotic morphological features, nuclear fragmentation 
and condensation. These results indicate that CD40-mediated signals might link to the caspase-mediated apoptosis pathway. Similar results were observed using anti-Fas antibody instead of the anti-CD40 antibody.

Effect of CD40 on $N F-\kappa B$ activation. Next, we investigated the

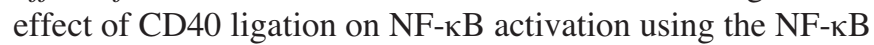
luciferase reporter gene assay, since NF- $\mathrm{KB}$ plays an important role in hepatocyte survival. NF- $\kappa \mathrm{B}$-fold induction increased in a dose-dependent manner to the anti-CD40 antibody. These results indicate that the $\mathrm{CD} 40$-mediated signal was linked to NF-кB (Fig. 5).

\section{Discussion}

CD40 belongs to the TNFR superfamily, whose members share sequence similarities in their extracellular domains that contain multiple cystein-rich pseudorepeats, and function to regulate cell growth and cell death (3). The expression of CD40 in human solid malignant tissues has been investigated. These reports demonstrated that six HCC cell lines constitutively expressed CD40 mRNA and membrane bound CD40 antigen, which was upregulated slightly by interferon- $\gamma(8,13)$. In addition, $60 \%$ of human HCC tissues demonstrated positive staining for CD40. These results suggest there is a relationship between CD40 expression and hepatocarcinogenesis. However in chronic liver disease, hepatocyte CD40 expression has not been well established. The present study is the first to demonstrate CD40 expression and function in a series of $\mathrm{HCV}$-associated chronic liver diseases.

CD40 positive hepatocytes were observed especially in the periportal areas with inflammation. In both the periportal and lobular areas, CD40 staining intensity became significantly stronger, correlating with the histological grading. The expression level of CD40 is known to be under the control of various cytokines. In particular, IFN- $\gamma$, interleukin $1 \beta$, and TNF- $\alpha$ enhance the expression of CD40 on monocytes (14), endothelial cells $(15,16)$, and thymic epithelium $(17)$. We previously found, following incubation with interferon- $\gamma$, that the CD40 levels of the HCC cell lines were enhanced from $2 \%$ to $50 \%$. In chronic hepatitis, several inflammatory cytokines are produced by infiltrating mononuclear cells, which exist particularly in portal and periportal areas. Our findings suggest hepatocyte CD40 expression is regulated by these cytokines, since CD40 expression correlates with inflammatory grades rather than fibrosis grades. CD40 expression and function might also be regulated by posttranscriptional and post-translational mechanisms. Thus, alternative splicing has been shown to generate multiple CD40 isoforms that are regulated differentially in activated macrophages and dendritic cells (DCs) (7). Through CD40 these isoforms can inhibit signaling and are also present in carcinoma cells. However, further study is needed to elucidate the regulatory mechanism of CD40 expression in hepatocytes.

Surprisingly our immunohistochemical study showed CD40 staining in both hepatocyte cytoplasm and cell membrane. The localized cytoplasmic CD40 is not thought to be a splicing form or deletion mutant of membrane-bound CD40, since CD40 mRNA of HCC cell lines showed no change in size by
RT-PCR methods (8). Similar cytoplasmic localization of CD40 was observed in malignant melanoma and HCCs (18). We have speculated that the occurrence of structural abnormalities is due to altered protein maturation by perturbation through post-translational modifications.

The CD40 expression and function besides B cells have not been well studied. Although CD40 stimulation can enhance Fas-mediated apoptosis in the highly CD40-positive myeloma cell line XG2 (19) and breast cancer cell line (20), it can otherwise inhibit Fas-mediated apoptosis in human dendritic cells (21), bladder carcinoma cell lines and HCCs (22). The evidence suggests that the CD40 signal may have a dual effect of both cell survival and death, depending on cellular characters or conditions. We have previously shown that in HepG2 cells, CD40 stimulation inhibited not only anti-Fas antibody-induced but also TNF- $\alpha$-induced apoptosis. We used actinomycin D (RNA synthesis inhibitor) to sensitize HepG2 cells to Fas and TNF receptor-mediated apoptosis; however, we observed a similar effect of CD40 stimulation on receptormediated apoptosis, confirming that this effect was prevalent in HepG2 cells. Our results indicate that CD40 signaling may be linked to the apoptotic pathway. A previous study showed that CD40 signal is also mediated by the TNFR associated factor (TRAF) family $(23,24)$. The $\mathrm{N}$-terminal domains of TRAF2 and TRAF3 proteins contain a series of five zinc finger

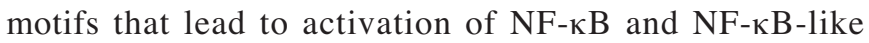
transcription factor complexes (23). It was also demonstrated that NF- $\mathrm{KB}$ suppressed the initiation of caspase activation by blocking the activation of caspase- 8 , suggesting that NF- $\mathrm{\kappa B}$ is activated by the CD40 signal and inhibits Fas or TNFRmediated apoptosis (25). As the exact mechanism of the apoptotic effect by CD40 stimulation was not well elucidated, we investigated $\mathrm{NF}-\kappa \mathrm{B}$ regulation by CD40 using the luciferase assay and revealed the CD40 activates NF- $\mathrm{\kappa B}$ in a dose-dependent manner. $\mathrm{NF}-\kappa \mathrm{B}$ is related to cell survival and has anti-apoptotic effects. These results indicate that CD40 expression in hepatocytes may contribute to the action of cell survival. Based on our results, CD40 can regulate both cell survival and death signaling.

Hepatocyte apoptosis can often be found in HCVassociated chronic liver disease. However, the regulatory mechanism of cell death in heptocytes is complicated and has not been well established. Similarly to CD40, the Fas antigen is also expressed at higher level in liver tissue with active inflammation (4). These results indicate Fas plays a significant role in the inflammatory and cell death pathways in chronic liver diseases. As for these receptor ligands, FasL is mainly expressed on cytotoxic $\mathrm{T}$ cells following an encounter with antigens or mitogens; while at the same time, CD40 ligand (CD40L) is expressed on activated CD4 ${ }^{+} \mathrm{T}$ cells. Thus, it is conceivable that hepatocytes are exposed to these ligands simultaneously during the $\mathrm{T}$ cell defense process.

These results suggest CD40 expression might play an important role in hepatocyte survival and death in HCVassociated chronic liver disease. Further study is critical in order to elucidate the mechanisms for these TNF receptormediated signals.

\section{References}

1. Nagata S: Apoptosis by death factor. Cell 88: 355-365, 1997. 
2. Doherty PC: Cell-mediated cytotoxicity. Cell 75: 607-612, 1993.

3. Nagata S and Golstein P: The Fas death factor. Science 267: 1449-1455, 1995.

4. Hiramatsu N, Hayashi N, Katayama K, Mochizuki K, Kawanishi Y, Kasahara A, Fusamoto H and Kamada T: Immunohistochemical detection of Fas antigen in liver tissue of patients with chronic hepatitis C. Hepatology 19: 1354-1359, 1994.

5. Stamenkovik I, Clark EA and Seed B: A B-lymphocyte activation molecule related to the nerve growth factor receptor and induced cytokines in carcinoma. EMBO J 8: 1403-1410, 1989.

6. Liu YJ, Joshua DE, Williams GT, Smith CA, Gordon J and MacLennan IC: Mechanism of antigen-driven selection in germinal centres. Nature 342: 929-931, 1989.

7. Eliopoulos AG and Young LS: The role of the CD40 pathway in the pathogenesis and treatment of cancer. Curr Opin Pharmacol 4: 360-367, 2004.

8. Sugimoto K, Shiraki K, Ito T, Fujikawa K, Takase K, Tameda Y, Moriyama M and Nakano T: Expression of functional CD40 in human hepatocellular carcinoma. Hepatology 30: 920-926, 1999.

9. Tong AW and Stone MJ: Prospects for CD40-directed experimental therapy of human cancer. Cancer Gene Ther 10: 1-13, 2003.

10. Dallman C, Johnson PW and Packham G: Differential regulation of cell survival by CD40. Apoptosis 8: 45-53, 2003.

11. Schattner EJ, Mascarenhas J, Bishop J, Yoo DH, Chadburn A, Crow MK and Friedman SM: CD4 ${ }^{+}$T-cell induction of Fasmediated apoptosis in Burkitt's lymphoma B cells. Blood 88: 1375-1382, 1996

12. Marches R, Racila E, Tucker TF, Picker L, Mongini P, Hsueh R, Vitetta ES, Scheuermann RH and Uhr JW: Tumour dormancy and cell signalling-III: role of hypercrosslinking of $\operatorname{IgM}$ and CD40 on the induction of cell cycle arrest and apoptosis in B lymphoma cells. Ther Immunol 2: 125-136, 1995.

13. Holub M, Zakeri SM, Lichtenberger C, Pammer J, Paolini P, Leifeld L, Rockenschaub S, Wolschek MF, Steger G, Gangl A, Willheim $\mathrm{M}$ and Reinisch W: Heterogeneous expression and regulation of CD40 in human hepatocellular carcinoma. Eur J Gastroenterol Hepatol 15: 119-126, 2003.

14. Alderson BMR, Armitage RJ, Tough TW, Strockbine L, Fanslow WC and Spriggs MK: CD40 expression by human monocytes: regulation by cytokines and activation of monocytes by the ligand for CD40. J Exp Med 178: 669-674, 1993.
15. Karmann K, Hughes CCW, Schechner J, Fanslow WC and Pober JS: CD40 on human endothelial cells: inducibility by cytokines and functional regulation of adhesion molecule expression. Proc Natl Acad Sci USA 92: 4342-4346, 1995

16. Kluth B, Hess S, Engelmann H, Schafnitzel S, Riethmüller G and Freucht H: Endothelial expression of CD40 in renal cell carcinoma. Cancer Res 57: 891-899, 1997.

17. Galy AH and Spits H: CD40 is functionally expressed on human thymic epithelial cells. J Immunol 149: 775-782, 1992.

18. Van den Oord JJ, Maes A, Stas M, Nuyts J, Battocchio S, Kasran A, Garmyn M, De Wever I and De Wolf-Peeters C: CD40 is a prognostic marker in primary cutaneous malignant melanoma. Am J Pathol 149: 1953-1961, 1996.

19. Bergamo A, Bataille R and Pellat-Deceunynck C: CD40 and CD95 induce programmed cell death in the human myeloma cell line XG2. Br J Haematol 97: 652-655, 1997.

20. Wingett DG, Vestal RE, Forcier K, Hadjokas N and Nielson CP. CD40 is functionally expressed on human breast carcinomas: Variable inducibility by cytokines and enhancement of Fasmediated apoptosis. Breast Cancer Res Treat 50: 27-36, 1998.

21. Björck P, Banchereau J and Flores-Romo L: CD40 ligation counteracts Fas-induced apoptosis of human dendritic cells. Int Immunol 9: 365-372, 1997

22. Jakobson E, Jönsson G, Björck P and Paulie S: Stimulation of CD40 in human bladder carcinoma cells inhibits anti-Fas/ APO-1 (CD95)-induced apoptosis. Int J Cancer 77: 849-853, 1998.

23. Rothe M, Sarma V, Dixit VM and Goeddel DD: TRAF2mediated activation of NF- $\mathrm{KB}$ by TNF receptor 2 and CD40. Science 269: 1424-1426, 1995.

24. Chaudhuri A, Orme S, Vo T. Wang W and Cherayil BJ: CD40mediated signals inhibit the binding of TNF receptor-associated factor 2 to the CD40 cytoplasmic domain. J Immunol 159: 4244-4251, 1997.

25. Wang CY, Mayo MW, Korneluk RG, Goeddel DV and Baldwin AS: NF-кB antiapoptosis: induction of TRAF1 and TRAF2 and c-IAP1 and c-IAP2 to suppress caspase-8 activation. Science 281: 1680-1683, 1998 . 